\title{
Associations of ABC (Hemoglobin A1c, Blood Pressure and Low-Density Lipoprotein Cholesterol) Goal Achievement With Chronic Kidney Disease in Type 2 Diabetic Patients With Preserved Kidney Function
}

\author{
Satomi Minato ${ }^{\mathrm{a}, \mathrm{b}}$, Akiko Takenouchic, Kaori Kitaoka ${ }^{\mathrm{a}, \mathrm{d}}$, Mika Takeuchi ${ }^{\mathrm{a}, \mathrm{e}}$, Ayaka Tsuboi ${ }^{\mathrm{a}, \mathrm{f}}$, \\ Miki Kurata ${ }^{\text {a, c }}$, Keisuke Fukuo a, c, Tsutomu Kazumia, g, h
}

\begin{abstract}
Background: We investigated cross-sectional and prospective associations of ABC (hemoglobin A1c (HbA1c), blood pressure and lowdensity lipoprotein cholesterol) goal attainment with chronic kidney disease. Cross-sectional association with carotid intima-media thickness (IMT) was evaluated as well.

Methods: Prevalence of low estimated glomerular filtration rate (eGFR $<60 \mathrm{~mL} / \mathrm{min} / 1.73 \mathrm{~m}^{2}$ ) and albuminuria (urinary albumin-tocreatinine ratio $(\mathrm{ACR}) \geq 30 \mathrm{mg} / \mathrm{g}$ ) were assessed at baseline and after a median follow-up of 6.0 years in 168 patients with type 2 diabetes with preserved kidney function (aged 62.3 years, 53.6\% men). Carotid IMT was measured at baseline only.
\end{abstract}

Results: At baseline, 47 (28.0\%), 45 (26.8\%), 63 (37.5\%) and 13 (7.7\%) patients achieved triple-goal, dual-goal, single-goal and nogoal, respectively. Achieving more $\mathrm{ABC}$ targets was associated with lower $\log$ ACR $(\mathrm{P}<0.01)$, lower percentage of albuminuria $(\mathrm{P}=$ $0.02)$, and lower carotid IMT $(\mathrm{P}<0.01)$ at baseline. Over 6.0 years, eGFR decreased from $76 \pm 16$ to $67 \pm 18 \mathrm{~mL} / \mathrm{min} / 1.73 \mathrm{~m}^{2}(\mathrm{P}<0.01)$ whereas ACR levels did not change. There were 32 patients with in-

Manuscript submitted September 16, 2019, accepted October 15, 2019

aResearch Institute for Nutrition Sciences, Mukogawa Women's University, Nishinomiya, Hyogo, Japan

${ }^{b}$ Graduate School of Human Science and Environment, University of Hyogo, Himeji, Hyogo, Japan

'Department of Food Sciences and Nutrition, School of Human Environmental Sciences, Mukogawa Women's University, Nishinomiya, Hyogo, Japan

${ }^{\mathrm{d}}$ Department of Public Health, Shiga University of Medical Science, Otsu, Shiga, Japan

eDepartment of Food Sciences and Nutrition, Faculty of Human Life and Environmental Sciences, Nagoya Women's University, Nagoya, Japan

fDepartment of Nutrition, Osaka City Juso Hospital, Osaka, Japan

gDiabetes Division, Department of Medicine, Kohnan Kakogawa Hospital, Kakogawa, Hyogo, Japan

${ }^{\mathrm{h}}$ Corresponding Author: Tsutomu Kazumi, Research Institute for Nutrition Sciences, Mukogawa Women's University, 6-46, Ikebiraki-cho, Nishinomiya, Hyogo 663-8558, Japan. Email: kazumi@mukogawa-u.ac.jp

doi: https://doi.org/10.14740/jocmr4001 cident reduced eGFR, eight with GFR stage progression, 15 with progression of albuminuric stages and five with doubling of ACR within the microalbuminuric range. Achieving more $\mathrm{ABC}$ targets decreased the percentage of deterioration of GFR stages $(30.8 \%, 28.6 \%, 24.4 \%$ and $14.9 \%$, respectively, $\mathrm{P}=0.01)$. Achieving two or more $(8.9 \%$ and $8.5 \%$, respectively) compared with one or less $\mathrm{ABC}$ targets $(15.4 \%$ and $15.9 \%$, respectively) was associated with less deterioration of albuminuria ( $\mathrm{P}<0.001)$. Although achieving more $\mathrm{ABC}$ targets was associated with lower annual decline in eGFR, the difference was not significant.

Conclusions: ABC goal achievement has shown cross-sectional and prospective associations with deterioration of chronic kidney disease in type 2 diabetic patients with preserved kidney function. Cross-sectional association with carotid IMT has been demonstrated as well. Reaching more $\mathrm{ABC}$ treatment targets may be important for preventing adverse renal outcomes.

Keywords: HbA1c; Blood pressure; LDL cholesterol; Albuminuria; Glomerular filtration rate

\section{Introduction}

The major objectives of diabetes management are to prevent development or progression of diabetic complications and disease conditions associated with diabetes, and to enable affected individuals to maintain their quality of life and life expectancy comparable to those seen in healthy individuals [1]. Intensive control for hemoglobin A1c (HbAlc), blood pressure (BP), and low-density lipoprotein cholesterol (LDL-C) have been shown to reduce cardiovascular disease (CVD) and death [24]. Japan Diabetes Society guidelines recommend that adults with diabetes maintain $\mathrm{HbAlc}<7 \%$, systolic and diastolic BP $<130 / 80 \mathrm{~mm} \mathrm{Hg}$ and LDL-C $<120 \mathrm{mg} / \mathrm{dL}$ [1], known as the $\mathrm{ABC}$ diabetes control. American Diabetes Association guidelines defined somewhat different $\mathrm{ABC}$ targets $(\mathrm{HbA} 1 \mathrm{c}<7.0 \%$, $\mathrm{BP}<140 / 90 \mathrm{~mm} \mathrm{Hg}$, and LDL-C $<100 \mathrm{mg} / \mathrm{dL}$ ) [5].

Chronic kidney disease (CKD) in diabetes is defined as elevated urinary albumin-to-creatinine ratio (ACR, $\geq 30 \mathrm{mg} / \mathrm{g}$ ) 
and/or low estimated glomerular filtration rate $(\mathrm{eGFR},<60$ $\left.\mathrm{mL} / \mathrm{min} / 1.73 \mathrm{~m}^{2}\right)[6,7]$. It is well recognized that patients with decreased GFR are at a greater risk for incident CVD and cardiovascular death. Type 2 diabetic patients with microalbuminuria or more advanced CKD are at extremely high risk of CVD and end-stage renal disease. Recently, the Trial to Reduce Cardiovascular Events with Aranesp Therapy has demonstrated that in type 2 diabetic patients with CKD and anemia, history of coronary artery disease was associated with progression to dialysis [8]. Thus, "there is a bidirectional relationship between reduced GFR and CVD, and the magnitude of the problem is underappreciated" [9]. Japanese Society of Nephrology recommended the same ABC targets as Japan Diabetes Society guidelines in "Clinical Practice Guidebook for Diagnosis and Treatment of Chronic Kidney Disease in 2012" [10]. However, studies which evaluate associations between ABC goal achievement and CKD in diabetes are limited. In the present study, therefore, we investigated cross-sectional and prospective associations of $\mathrm{ABC}$ goal attainment with $\mathrm{CKD}$ in patients with type 2 diabetes with preserved kidney function. Cross-sectional association with carotid intima-media thickness (IMT), a surrogate marker of subclinical atherosclerosis [11], was evaluated as well.

\section{Patients and Methods}

We studied 168 patients with type 2 diabetes (aged 62.3 years, $53.6 \%$ men), whose details have been reported elsewhere [12]. They had been regularly (once a month) attending the clinic in 2004 and 2005. They were enrolled at the first visit in 2005 and followed up for the subsequent at least 24 months through December 31, 2012 to assess kidney function with a median follow-up of 6.0 years (interquartile range: 4.1 - 6.5 years). Data on age, duration of diabetes and drugs currently being taken were collected at the entry of the study. We excluded patients with serum creatinine $\geq 2.0 \mathrm{mg} / \mathrm{dL}$, with hepatitis B surface antigen or antibodies against hepatitis $C$ virus, and with aspartate aminotransferase and alanine aminotransferase of $100 \mathrm{U} / \mathrm{L}$ or greater. Study protocol was consistent with the Japanese Government's Ethical Guidelines Regarding Epidemiological Studies in accordance with the Declaration of Helsinki.

Waist circumference, weight and BP were measured by registered nurses on each monthly visit. As previously reported in detail [12-14], blood was withdrawn on two occasions; at 2 $\mathrm{h}$ after breakfast taken at home and after an overnight fasting in 153 patients (91\%). This was done every other month. In the remaining 15 patients, blood was withdrawn after an overnight fasting. Plasma glucose (PG), serum total cholesterol, triglycerides, high-density lipoprotein cholesterol (HDL-C) and uric acid were measured by standard methods using an autoanalyzer. $\mathrm{HbA} 1 \mathrm{C}$ values were determined by high performance liquid chromatography. LDL-C was calculated by Friedewald's formula using lipid levels obtained in blood samples taken after an overnight fasting.

Intrapersonal means of body mass index (BMI), waist circumference, systolic and diastolic BP, HbA1c, serum cholesterol and HDL-C were calculated based on 12 measurements in 168 patients. Intrapersonal means of LDL-C were calculated based on six measurements in 153 patients and 12 measurements in the remaining 15 patients. Mean levels of HbA1c, systolic and diastolic BP and LDL-C based on 6 - 12 measurements over a 12-month period were used to evaluate goal achievement of ABC.

Urinary albumin was measured in 161 patients [13] at the entry and the end of the observation time period using a turbidimetric immunoassay in random urine samples. Normoalbuminuria, microalbuminuria and macroalbuminuria were defined as ACR < $30 \mathrm{mg} / \mathrm{g}$, ACR between 30 and $299 \mathrm{mg} / \mathrm{g}$ and ACR $\geq 300 \mathrm{mg} / \mathrm{g}$, respectively $[5,6]$. Progression of albuminuria stages and doubling of ACR within the microalbuminuric range were defined as deterioration of albuminuria.

Serum and urinary creatinine were measured enzymatically and eGFR was determined using the equation recommended by the Japanese Society for Nephrology [15]. Patients were staged according to the level of eGFR in $\mathrm{mL} / \mathrm{min} / 1.73 \mathrm{~m}^{2} ; \mathrm{G} 1:>90$, G2: 60 - 89, G3a: 45 - 59, G3b: 30 - 44, G4: 15 - 29 [6]. CKD in diabetes is defined as albuminuria (ACR $\geq 30 \mathrm{mg} / \mathrm{g}$ ) and/or low eGFR $\left(<60 \mathrm{~mL} / \mathrm{min} / 1.73 \mathrm{~m}^{2}\right)[6,7]$. Incident reduced eGFR was defined as eGFR $<60 \mathrm{~mL} / \mathrm{min} / 1.73 \mathrm{~m}^{2}$ in participants with an eGFR $>60 \mathrm{~mL} / \mathrm{min} / 1.73 \mathrm{~m}^{2}$ at baseline. Annual changes in eGFR were estimated using linear regression with a median of 52 creatinine measurements (interquartile range: 31 - 60) over 6.0 years of follow-up in each patient $[11,12]$.

Carotid IMT was measured in 162 patients at baseline as previously described [16]. This was done by carotid artery ultrasonography (Shimadzu SDU-2200, Shimadzu, Tokyo, Japan). Carotid IMT was evaluated by maximum and average evaluations. The former was obtained by the IMT value at a maximal point of the region. The latter is the average value of 250 computer-based points in the region. Mean values of the right and left maximum IMT and mean IMT were used for statistical analysis.

Data were presented as mean \pm standard error (SE) unless otherwise stated. Associations of continuous variables and frequencies of conditions with the number of $\mathrm{ABC}$ goals attained and $\mathrm{P}$ trend were derived using Jonckheere-Terpstra test and Cochran-Armitage trend analysis, respectively. A two-tailed P $<0.05$ was considered statistically significant. All calculations were performed with SPSS system 15.0 (SPSS Inc., Chicago, IL).

\section{Results}

\section{Patients' characteristics}

Among 168 patients, $115(68.5 \%)$ were on oral anti-diabetes drugs or insulin, and 87 patients $(51.5 \%)$ were on BP-lowering drugs [12-14]. Sixty-three patients $(37.3 \%)$ received statin or ezetimibe while only $12(7.1 \%)$ and 11 patients $(6.5 \%)$ received fibrates and allopurinol, respectively. Their mean $\mathrm{HbA1C}$, BP and LDL-C were 7.0\%, 128/72 $\mathrm{mm} \mathrm{Hg}$, and $111 \mathrm{mg} / \mathrm{dL}$, respectively. The prevalence of meeting ABC goal were $54.8 \%$ $(\mathrm{n}=92), 54.2 \%(\mathrm{n}=91)$ and $66.1 \%(\mathrm{n}=111)$, respectively.

Of 168 patients, $47(28.0 \%)$ achieved triple-goal while 13 (7.7\%) achieved no-goal (Table 1). Dual-goal and single-goal 
Table 1. Anthropometric and Clinical Characteristics of Patients at Baseline According to the Number of Goal Achievement

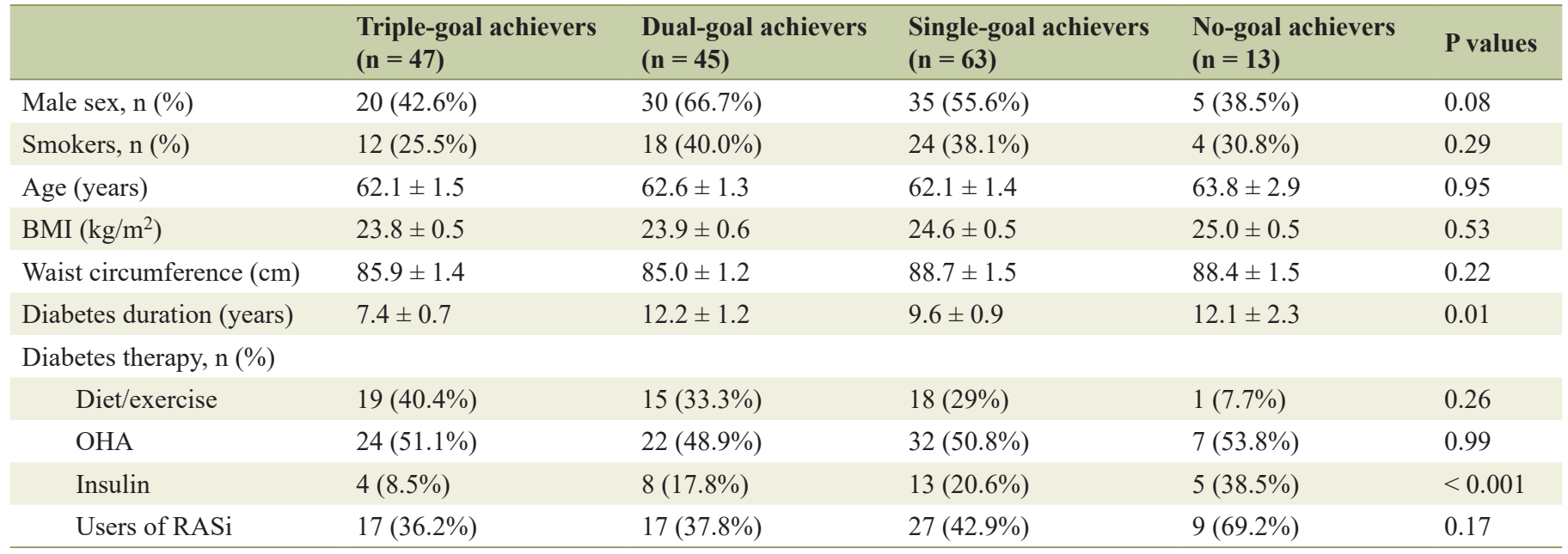

Mean \pm SE or $n(\%)$. SE: standard error; OHA: oral anti-diabetes drugs; RASi: renin-angiotensin-aldosterone system inhibitors; BMI: body mass index.

attainment was found in $45(26.8 \%)$ and 63 patients $(37.5 \%)$, respectively. The proportion of men was higher in single- and dual-goal compared with no-goal and triple-goal achievers. Triple-goal compared with no-goal achievers had shorter diabetes duration and lower percentage of users of insulin and BP-lowering drugs. The proportion of men was highest in dual-goal achievers, followed by single- and then triple-goal achievers and then lowest in no-goal achievers. However, four groups did not differ in age, anthropometric indices and the proportion of users of oral anti-diabetes drugs, statin or ezetimibe and fibrates and smokers.

As the number of goals attained decreased, HDL-C decreased while fasting triglyceride and uric acid did not change
(Table 2). At baseline, eGFR averaged $76 \pm 1 \mathrm{~mL} / \mathrm{min} / 1.73 \mathrm{~m}^{2}$, and 27 of 168 patients (16.0\%) had reduced eGFR. Microalbuminuria and macroalbuminuria were found in 45 (26.8\%), and seven patients $(4.2 \%)$, respectively, at baseline.

\section{Cross-sectional study}

At baseline, achieving more goals was associated with lower $\log \mathrm{ACR}$ and lower percentage of albuminuria (ACR $\geq 30$ $\mathrm{mg} / \mathrm{g}$ ) (Fig. 1). However, there was no difference in eGFR and the percentage of patients with reduced eGFR among four groups (Table 2). Achieving more goals was also associated

Table 2. Glycemic, Lipids and Blood Pressure Control and Kidney Function of Patients at Baseline According to the Number of Goal Achievement

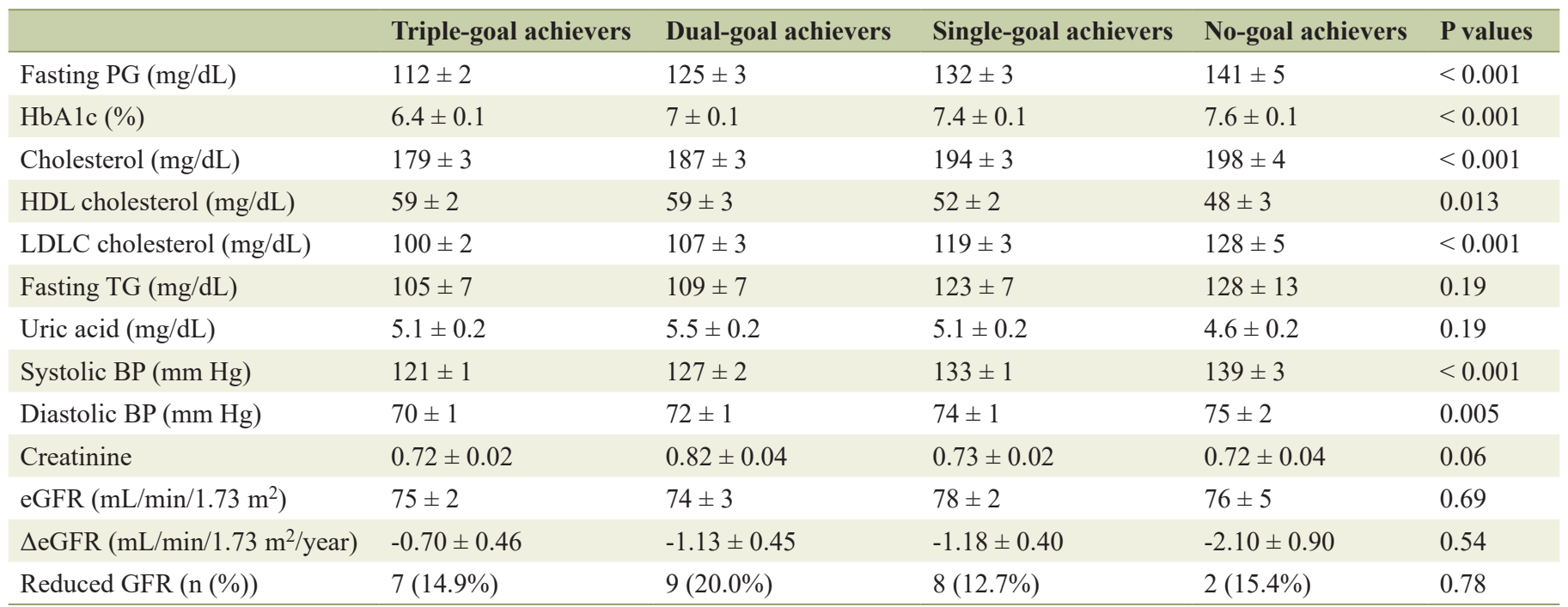

Mean \pm SE or $n(\%)$. SE: standard error; PG: plasma glucose; LDL-C: low-density lipoprotein cholesterol; TG: triglyceride: BP: blood pressure; eGFR: estimated glomerular filtration rate; $\triangle \mathrm{eGFR}$ : annual changes in eGFR. 

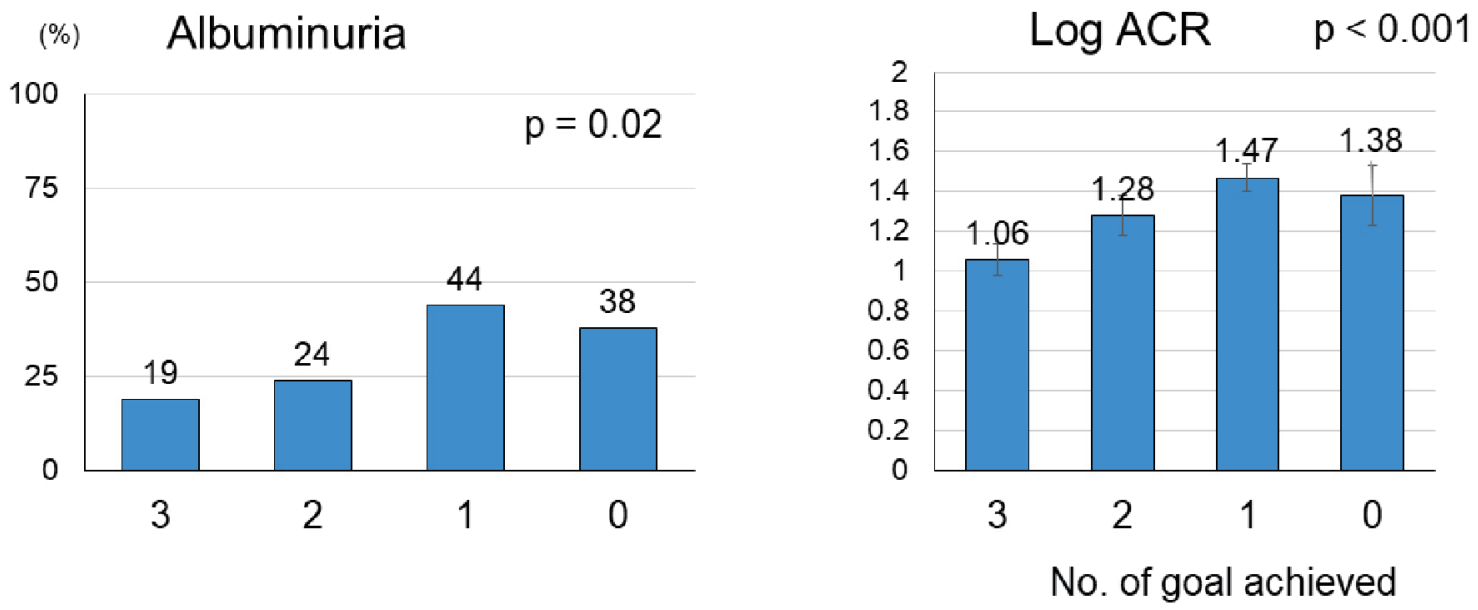

Figure 1. Mean ( \pm SE) log urinary $A C R$ and the percentage of patients with albuminuria $(A C R \geq 30 \mathrm{mg} / \mathrm{g})$ at baseline according to the number of ABC (HbA1c, blood pressure and LDL-C) goal achievement. Among 168 patients with type 2 diabetes, 47, 45, 63 and 13 patients achieved triple-goal, dual-goal, single-goal and no-goal, respectively, at baseline. SE: standard error; ACR: albumin-to-creatinine ratio; HbA1c: hemoglobin A1c; LDL-C: low-density lipoprotein cholesterol.

with a stepwise decrease in maximum carotid IMT as well as mean carotid IMT (Fig. 2).

\section{Prospective study}

After a median follow-up of 6.0 years, eGFR decreased from $76 \pm 1$ to $67 \pm 1 \mathrm{~mL} / \mathrm{min} / 1.73 \mathrm{~m}^{2}(\mathrm{P}<0.01)$ with an annual decline of $1.11 \pm 0.23 \mathrm{~mL} / \mathrm{min} / 1.73 \mathrm{~m}^{2}$. Although an annual decline of eGFR increased with a decreasing number of goals attained, differences were not significant (Table 2). Incident reduced eGFR and progression of GFR stages occurred in 32 and eight patients, respectively. The percentage of patients with deterioration of GFR stages was increased in a stepwise fashion with decreasing numbers of goal achievement (Fig. 3).

There was no difference in ACR levels between at base-

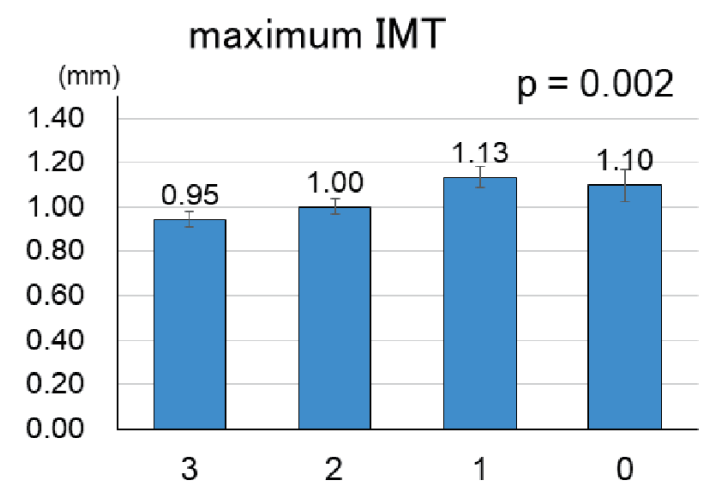

line and after a 6-year follow-up $(84 \pm 25$ and $87 \pm 26 \mathrm{mg} / \mathrm{g}$, respectively). Progression of albuminuria stages and doubling of ACR within the microalbuminuric range occurred in 15 and five patients, respectively, among 161 patients. Attainment of two or more targets at baseline was associated with benefits on reducing deterioration of albuminuric stages compared with those with one or less target achieved (Fig. 4).

\section{Discussion}

The present study has shown in a real-life situation that $\mathrm{ABC}$ achievement was associated cross-sectionally and prospectively with renal complications in type 2 diabetic patients with preserved kidney function. Cross-sectional association between $\mathrm{ABC}$ achievement and carotid IMT, a surrogate marker of sub-

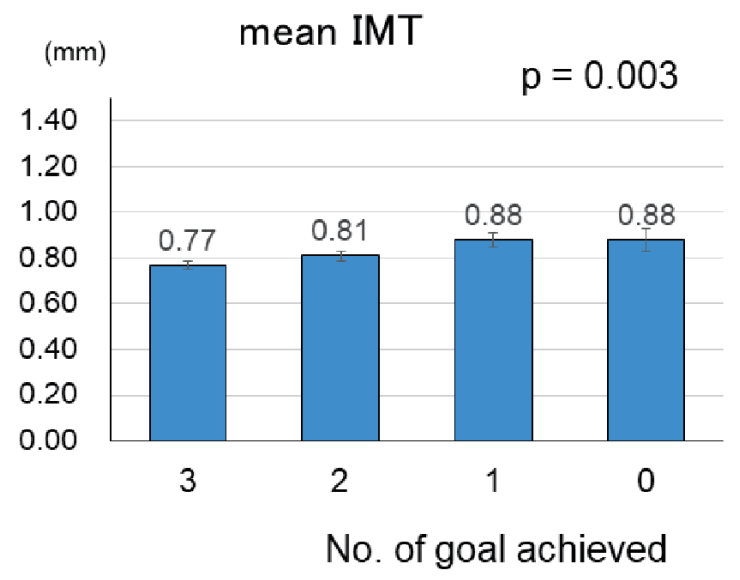

Figure 2. Maximum and mean carotid IMT at baseline according to the number of $A B C(\mathrm{HbA} 1 \mathrm{c}$, blood pressure and LDL-C) goal achievement (mean $\pm \mathrm{SE}$ ). See Figure 1 for the number of patients in each goal achievers. IMT: intima-media thickness; HbA1c: hemoglobin A1c; LDL-C: low-density lipoprotein cholesterol; SE: standard error. 


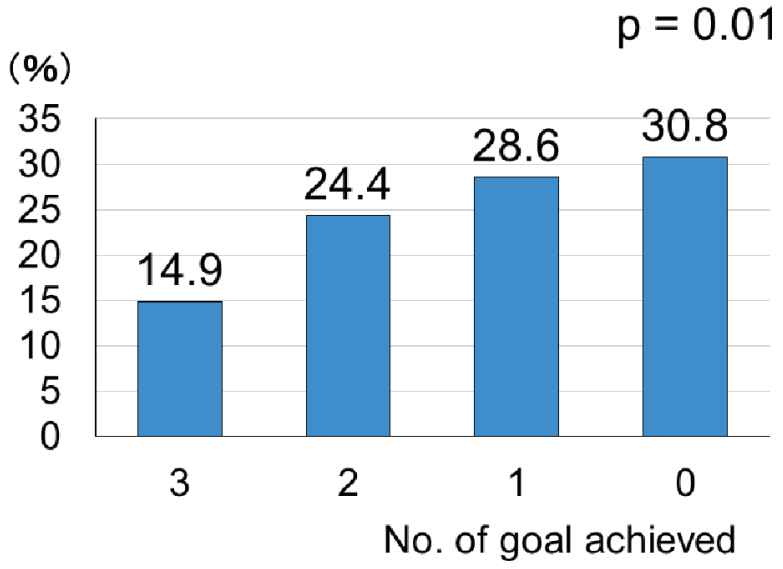

Figure 3. The percentage of deterioration of glomerular filtration rate stages over a median follow-up of 6.0 years according to the number of $\mathrm{ABC}(\mathrm{HbA} 1 \mathrm{c}$, blood pressure and LDL-C) goal achievement at baseline. See Figure 1 for the number of patients in each goal achievers. HbA1c: hemoglobin A1c; LDL-C: low-density lipoprotein cholesterol.

clinical atherosclerosis [11], at baseline in the current study may be in line with previous studies that $\mathrm{ABC}$ goal achievement was associated with macrovascular complications in type 2 diabetes patient [17-23].

Despite guidelines recommending multi-factorial treatment of cardiometabolic risk factors [1, 5, 10], studies which evaluate association between $\mathrm{ABC}$ goal achievement and microvascular complications in diabetes in a real-life situation are limited. Associations of $\mathrm{ABC}$ achievement with log ACR and the percentage of patients with albuminuria (ACR $\geq 30$ $\mathrm{mg} / \mathrm{g}$ ) at baseline in the present study appear to be consistent with cross-sectional and retrospective studies that triple-goal achievement was associated with better microvascular complications in patients with type 2 diabetes [22, 23].

Chan et al [24] compared effects of structured versus usual care on death and/or end-stage renal disease for 2 years in type 2 diabetes who had plasma creatinine levels of $150-350$ $\mu \mathrm{mol} / \mathrm{L}(1.70-4.00 \mathrm{mg} / \mathrm{dL})$. Treatment goals included ABC, triglycerides $<2 \mathrm{mmol} / \mathrm{L}$, and persistent treatment with reninangiotensin blockers. Attainment of $\geq 3$ treatment goals rather than structured care reduced end-stage renal disease and death. In the Coronary Artery Calcification in Type 1 Diabetes Study [25], 652 participants were 19 - 56 years old at baseline and reexamined 6-years later. Microvascular complications included incident albuminuria or rapid GFR decline (>3.3\%/year) and laser eye-therapy. Macrovascular complications were defined as coronary artery calcium progression, measured by electronbeam computed-tomography. $\mathrm{ABC}$ achievement at baseline strongly predicted microvascular but not macrovascular complications over 6 years [25]. In the present study on type 2 diabetes patients with preserved kidney function, $\mathrm{ABC}$ achievement at baseline predicted deterioration of albuminuria and GFR stages over 6 years in a real-life situation.

The strength of the current study is that mean levels of $\mathrm{ABC}$ used for the evaluation of goal achievement were those of 6 - 12 measurements within a 12-month period. In addition, decreased GFR was diagnosed based on 2 - 4 creatinine

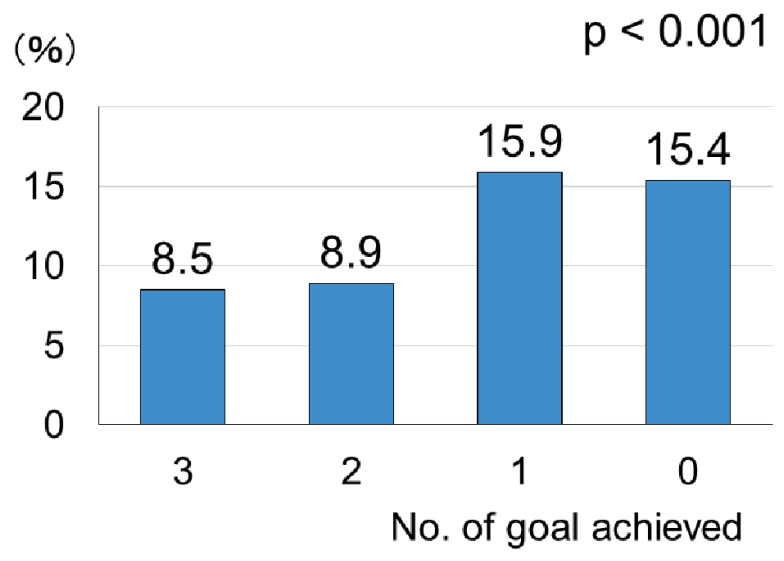

Figure 4. The percentage of deterioration of albuminuric stages over a median follow-up of 6.0 years according to the number of $A B C(\mathrm{HbA} 1 \mathrm{c}$, blood pressure and LDL-C) goal achievement at baseline. See Figure 1 for the number of patients in each goal achievers. HbA1c: hemoglobin A1c; LDL-C: low-density lipoprotein cholesterol.

measurements over a period of 3 months or longer not only at the entry of, but at the end of the observation. Further, as previously described in detail $[12,13]$, serum creatinine and hence eGFR during follow-up period were much more frequently measured. This could contribute to the reliability of changes in kidney function. Major limitations are that number of study participants was small and that the study was from a single clinic in Japan. However, our study participants had similar characteristics to those reported in a previous large-scale study in Japan [26]. We did not use precise measures of kidney function, like iothalamate clearance. In addition, we studied Japanese only, which limits the generalizability. Finally, the sample size and the number of CKD-related incidents occurred in the present study seem to be small to lead a robust conclusion although statistical power was not calculated.

\section{Conclusions}

$\mathrm{ABC}$ goal achievement prospectively predicted deterioration of CKD in type 2 diabetic patients with preserved kidney function in a real-life situation. Cross-sectional association with carotid IMT has been demonstrated as well. These data highlight the importance of achieving more $\mathrm{ABC}$ goals to prevent deterioration of CKD. Further studies are needed to confirm the association in other ethnic groups with more patients.

\section{Acknowledgments}

We are indebted to all the participants for their dedicated and conscientious collaboration.

\section{Financial Disclosure}

None to declare. 


\section{Conflict of Interest}

None to declare.

\section{Informed Consent}

Informed consents were obtained from all participants.

\section{Author Contributions}

SM, AT, KK, MT, AT and MK have made substantial contributions to acquisition, analysis and interpretation of the data. $\mathrm{KF}$ has been involved in drafting the manuscript. TK has been involved in revising it critically for important intellectual content; has given final approval of the version to be published; and agreed to be accountable for all aspects of the work in ensuring that questions related to the accuracy or integrity of any part of the work are appropriately investigated and resolved. All authors read and approved the final manuscript.

\section{Abbreviations}

ACR: albumin-to-creatinine ratio; BMI: body mass index; BP: blood pressure; CKD: chronic kidney disease; CVD: cardiovascular disease; eGFR: estimated glomerular filtration rate; HbA1c: hemoglobin A1c; HDL-C: high-density lipoprotein cholesterol; LDL-C: low-density lipoprotein cholesterol; IMT: intima-media thickness; PG: plasma glucose; TG: triglycerides

\section{References}

1. Tajima N, Noda M, Origasa H, Origasa H, Noda M, Ueki K, Tajima N. Evidence-based practice guideline for the treatment for diabetes in Japan 2013. Diabetol Int. 2015;6:151-87.

2. Ray KK, Seshasai SR, Wijesuriya S, Sivakumaran R, Nethercott S, Preiss D, Erqou S, et al. Effect of intensive control of glucose on cardiovascular outcomes and death in patients with diabetes mellitus: a meta-analysis of randomised controlled trials. Lancet. 2009;373(9677):17651772 .

3. Emdin CA, Rahimi K, Neal B, Callender T, Perkovic $\mathrm{V}$, Patel A. Blood pressure lowering in type 2 diabetes: a systematic review and meta-analysis. JAMA. 2015;313(6):603-615.

4. Cholesterol Treatment Trialists C, Kearney PM, Blackwell L, Collins R, Keech A, Simes J, Peto R, et al. Efficacy of cholesterol-lowering therapy in 18,686 people with diabetes in 14 randomised trials of statins: a metaanalysis. Lancet. 2008;371(9607):117-125.

5. American Diabetes Association. Standards of medical care in diabetes-2015. Diabetes Care. 2015;38(Suppl1):S1-94.

6. National Kidney Foundation. KDIGO 2012 clinical practice guideline for the evaluation and management of chronic kidney disease. Kidney Int. 2013;3(Suppl):1-150.

7. Tuttle KR, Bakris GL, Bilous RW, Chiang JL, de Boer IH, Goldstein-Fuchs J, Hirsch IB, et al. Diabetic kidney disease: a report from an ADA Consensus Conference. Diabetes Care. 2014;37(10):2864-2883.

8. Sabe MA, Claggett B, Burdmann EA, Desai AS, Ivanovich P, Kewalramani R, Lewis EF, et al. Coronary artery disease is a predictor of progression to dialysis in patients with chronic kidney disease, type 2 diabetes mellitus, and anemia: an analysis of the Trial to Reduce Cardiovascular Events with Aranesp Therapy (TREAT). J Am Heart Assoc. 2016;5(4):e002850.

9. Barrows IR, Raj DS. Janus Face of Coronary Artery Disease and Chronic Kidney Disease. J Am Heart Assoc. 2016;5(4):e003596.

10. Clinical practice guidebook for diagnosis and treatment of chronic kidney disease in 2012 (2012, in Japanese). www.jsn.or.jp.

11. Nezu T, Hosomi N, Aoki S, Matsumoto M. Carotid Intima-Media Thickness for Atherosclerosis. J Atheroscler Thromb. 2016;23(1):18-31.

12. Takenouchi A, Tsuboi A, Terazawa-Watanabe M, Kurata M, Fukuo K, Kazumi T. Direct association of visit-tovisit $\mathrm{HbA1c}$ variation with annual decline in estimated glomerular filtration rate in patients with type 2 diabetes. J Diabetes Metab Disord. 2015;14:69.

13. Takenouchi A, Tsuboi A, Kurata M, Fukuo K, Kazumi T. Carotid Intima-Media Thickness and Visit-to-Visit HbAlc Variability Predict Progression of Chronic Kidney Disease in Type 2 Diabetic Patients with Preserved Kidney Function. J Diabetes Res. 2016;2016:3295747.

14. Takenouchi A, Tsuboi A, Kitaoka K, Minato S, Kurata M, Fukuo K, Kazumi T. Visit-to-Visit Low-Density Lipoprotein Cholesterol Variability Is an Independent Determinant of Carotid Intima-Media Thickness in Patients With Type 2 Diabetes. J Clin Med Res. 2017;9(4):310-316.

15. Matsuo S, Imai E, Horio M, Yasuda Y, Tomita K, Nitta $K$, Yamagata $K$, et al. Revised equations for estimated GFR from serum creatinine in Japan. Am J Kidney Dis. 2009;53(6):982-992.

16. Yanase T, Nasu S, Mukuta Y, Shimizu Y, Nishihara T, Okabe T, Nomura M, et al. Evaluation of a new carotid intima-media thickness measurement by B-mode ultrasonography using an innovative measurement software, intimascope. Am J Hypertens. 2006;19(12):1206-1212.

17. Wong ND, Zhao Y, Patel R, Patao C, Malik S, Bertoni AG, Correa A, et al. Cardiovascular risk factor targets and cardiovascular disease event risk in diabetes: a pooling project of the atherosclerosis risk in communities study, multi-ethnic study of atherosclerosis, and Jackson heart study. Diabetes Care. 2016;39(5):668-676.

18. Vazquez-Benitez G, Desai JR, Xu S, Goodrich GK, Schroeder EB, Nichols GA, Segal J, et al. Preventable major cardiovascular events associated with uncontrolled glucose, blood pressure, and lipids and active smoking in adults with diabetes with and without cardiovascular disease: a contemporary analysis. Diabetes Care. 2015;38(5):905-912.

19. Wan EYF, Fung CSC, Yu EYT, Chin WY, Fong DYT, 
Chan AKC, Lam CLK. Effect of multifactorial treatment targets and relative importance of hemoglobin a1c, blood pressure, and low-density lipoprotein-cholesterol on cardiovascular diseases in Chinese primary care patients with type 2 diabetes mellitus: a population-based retrospective cohort study. J Am Heart Assoc. 2017;6(8):e006400.

20. Kong AP, Yang X, Ko GT, So WY, Chan WB, Ma RC, $\mathrm{Ng} \mathrm{VW}$, et al. Effects of treatment targets on subsequent cardiovascular events in Chinese patients with type 2 diabetes. Diabetes Care. 2007;30(4):953-959.

21. Nichols GA, Joshua-Gotlib S, Parasuraman S. Independent contribution of $\mathrm{A} 1 \mathrm{C}$, systolic blood pressure, and LDL cholesterol control to risk of cardiovascular disease hospitalizations in type 2 diabetes: an observational cohort study. J Gen Intern Med. 2013;28(5):691-697.

22. Yokoyama H, Oishi M, Takamura H, Yamasaki K, Shirabe SI, Uchida D, Sugimoto $H$, et al. Large-scale survey of rates of achieving targets for blood glucose, blood pressure, and lipids and prevalence of complications in type 2 diabetes (JDDM 40). BMJ Open Diabetes Res Care. 2016;4(1):e000294.
23. Shi Q, Liu S, Krousel-Wood M, Shao H, Fonseca V, Shi L. Long-term outcomes associated with triple-goal achievement in patients with type 2 diabetes mellitus (T2DM). Diabetes Res Clin Pract. 2018;140:45-54.

24. Chan JC, So WY, Yeung CY, Ko GT, Lau IT, Tsang MW, Lau KP, et al. Effects of structured versus usual care on renal endpoint in type 2 diabetes: the SURE study: a randomized multicenter translational study. Diabetes Care. 2009;32(6):977-982.

25. Bjornstad P, Maahs DM, Rewers M, Johnson RJ, SnellBergeon JK. ABC goal achievement predicts microvascular but not macrovascular complications over 6-years in adults with type 1 diabetes: the Coronary Artery Calcification in Type 1 Diabetes Study. J Diabetes Complications. 2014;28(6):762-766.

26. Sone H, Tanaka S, Iimuro S, Tanaka S, Oida K, Yamasaki Y, Oikawa S, et al. Long-term lifestyle intervention lowers the incidence of stroke in Japanese patients with type 2 diabetes: a nationwide multicentre randomised controlled trial (the Japan Diabetes Complications Study). Diabetologia. 2010;53(3):419-428. 